\title{
Paying for cancer care-a new dilemma
}

Karol Sikora

$J$ R Soc Med 2007;100:166-169

NHS spending is now at unprecedented levels. Over $£ 90$ billion is being spent this year alone and by 2008 this will reach $£ 105$ billion. Yet the quality of service provision and access to technology still does not match that of our European neighbours. Increasing the total budget spent on health is one solution. This can either come from increased taxation or by direct payment at the point of care: a copayment. Another is a radical reform of the entire system from its insurance function through to its delivery system. Stripping away bureaucracy to reduce costs is an essential component of the reform process. A recent report ${ }^{1}$ suggests that we are heading for a $£ 7$ billion deficit in the NHS by 2011 - and that's not allowing for step changes in technology. That represents an income tax increase of two pence in the pound for all those at work unless we use some other payment mechanism.

Cancer care costs are spiralling out of control in every healthcare environment. Aging populations with a wide range of medical problems are consuming vastly increasing amounts of care. New technology - drugs, devices and procedures - are powerful inflationary drivers in an information rich, consumer-oriented world. ${ }^{2}$ Different healthcare systems are using a variety of approaches to dampen demand. Rationing, both overt and covert, inevitably leads to inequity. Britain's NHS is undergoing a slow reform process, but huge variations exist in the way patients access its services, depending on their location, education and socio-economic background. There are also major differences in prioritizing services by those responsible for their payment (the Primary Care Trusts, often advised by the local Cancer Networks). A study in 2006 showed clearly that new cancer drug use in Britain was consistently and significantly well below that of our EU neighbours. ${ }^{3}$ Similarly, another comparative EU study on the availability of radiotherapy last year ranked the UK near the bottom, on the level with the accession countries of Central Europe, even after the recent purchase of over 100 machines using lottery money. ${ }^{4}$ An alliance of British cancer charities have successfully lobbied for a second cancer plan to improve services. Whilst this would be beneficial, there is no evidence that any additional tax-based NHS funding

Medical Director, CancerPartnersUK, 21 Barrett Street, London W14 1BD, UK E-mail: Karol.Sikora@CancerPartnersUK.org will be available for this laudable concept. Cancer is seen as a ticked box in Britain.

There is now evidence of a growing use of co-payments to break through the access barriers in the NHS. This applies to areas as diverse as implanted hearing aid devices, access to diagnostics such as MRI scans and home nursing care services. Politicians of all persuasions seem to be in denial about their existence and are reluctant to get involved in debate. Meanwhile cancer patients are beginning to develop sophisticated approaches to buying extra clinical services either from the NHS directly or through the selective use of the private sector to purchase upgrades to their basic NHS care.

With drugs the economics are relatively straightforward, but with precision radiotherapy the issues are more complex and so far hidden from the political media limelight. There are 61 radiotherapy centres in the UK. Of these, 28 now have equipment to provide intensity modulated radiotherapy, which is now standard in the US and most of Western Europe. Only three British centres, however, provide intensity modulated radiotherapy routinely to significant numbers of patients: Clatterbridge (Liverpool), Ipswich and the Royal Marsden (London). Staff and funding shortages are blamed. Savvy patients are beginning to travel long distances for more precise radical radiotherapy in order to suffer fewer long-term side effects. The imminent publication of the National Radiotherapy Advisory Group report, which shows wide variation in radiotherapy utilization across the country, is clearly going stimulate new models for radiotherapy provision. Furthermore, delays abound, with a three-month waiting time for radiotherapy being common in the UK. In contrast, a centre in Zurich, Switzerland, currently has a working group engaged in re-engineering the time from first contact to radiotherapy delivery from five to three working days.

\section{THE DEBATE}

Is it time to open the debate about whether the NHS should be able to provide not just a core package of services but to also allow patients to contribute financially for improved services for themselves and their families? This would reduce the inequity resulting from the current confusion where some patients have to receive all their care privately. This is simply unaffordable to many, who have, after all, paid their tax-based insurance premium to the NHS for the 
core package. Being open about the possibilities could lead to a set of innovative insurance products to pay for specific options. This could herald an era of real patient choice.

\section{OPTIONS FOR PAYING FOR CANCER TREATMENTS}

\section{Denial}

Denial of the existence of a drug, device or service and refusal to discuss any of its possible benefits during an NHS consultation is one way of dealing with the situation. This is not really possible in an age of patient empowerment and free availability of information on the internet. A recent Cancerbackup (http://www.cancerbackup.org.uk/Home) report shows that patients want all the information even if they cannot access all treatments. ${ }^{5}$ Denial is the ultimate in social solidarity: if a drug is not available for all on the NHS then none will receive it. It is the doctrine of medical communism.

\section{Transfer of entire care to the private sector}

After a full discussion of the options available the patient is referred for entirely private care by either the same consultant or by a colleague at a private hospital. Without insurance, this amounts to signing a blank cheque. Who knows what medical complications will occur that require expensive treatment? It's also hugely inequitable, as only those of substantial means can afford to do it and yet all have paid for the basic NHS component through taxation. This approach is apparently given out as a policy statement to patients in certain parts of Britain, although no official written documentation can be identified in the NHS.

\section{Obtain the upgrade only in the private sector}

After a full discussion of the options available the patient is referred for private care to either the same consultant or to a colleague at a private hospital just for the component of care not available through the NHS. This results in the patient having two oncologists and a difficulty in dealing with treatment-related complications: should they be treated freely on the NHS or with further payment in the private sector? This approach is of course fairer than making patients pay for all their care privately but increases the inconvenience for the patient, who must now receive their care in two places. It also could lead to confusion, as two separate sets of records are kept in different locations.

\section{NHS provision of upgraded drug with an invoice going to the patient}

After full discussion of the options available the patient receives the relevant upgrade within the same NHS unit. The patient is then invoiced by the NHS just for the extra treatment, together with a suitable mark-up to cover the hospital's costs and to improve the overall quality of care for all. Clinical care and information flow is seamless and any complications can be dealt with expeditiously. This strategy will of course lead to patients sitting in the same day ward receiving different drugs based solely on their ability to pay, but this is already happening where private and NHS patients are treated at the same unit.

\section{Leaving the patient to their own devices}

The patient is dissatisfied with the response and obtains the relevant upgrade from an internet pharmacy without informing their consultant. Edicts that ban the use of copayments to obtain drugs could drive underground markets where patients do not inform their NHS health professionals of their plans. This is clearly the most dangerous option. Certain complementary and alternative therapies already follow this pattern. Unscrupulous suppliers and service providers would undoubtedly emerge to meet this new market.

\section{ADVANCES DURING 2007}

There are huge changes taking place in cancer medicine which will significantly impact on the costs of the optimal care for this disease. The biggest financial impact will come from the registration of several high cost molecularly targeted drugs in adjuvant settings for common diseases such as lung, breast and colorectal cancer. The precedent of trastuzumab (Herceptin) is likely to be repeated for several other agents - such as bevacizumab (Avastin), erlotinib (Tarceva), lapatinib (Tykerb) and cetuximab (Erbitux)over the next 12 months. The strategy will be the same: initial licensing will be sought in the USA for metastatic cancer but this is likely to be followed rapidly in the EU and Japan as industry seeks global markets. Adjuvant studies will be pursued aggressively to bring these new drugs into the earlier phase of cancer management, dramatically increasing sales. Molecularly targeted therapies have incurred huge research and development expenditures by big pharma and therefore are expected to be future blockbusters. These include both small molecules which are taken as oral daily medication and monoclonal antibodies which are given as intravenous infusions every two or three weeks.

Primary Care Trusts will need to produce consistent advice as to what they will pay for in the adjuvant setting by simply awaiting NICE guidelines or taking independent advice on cost-benefit. This is a much more challenging area for rationing than first or second-line chemotherapy for metastatic cancer, as the overall survival benefits may much greater. High cost drugs are likely to create the most significant short term financial pressures in cancer care for the foreseeable future. The relevant agents and the 
Table 1 Cancer drug cost pressures for 2007. The yearly cost includes administration and medical care costs which are not normally included by manufacturers in their media communications. Life cycle management strategies will involve developing new indications for these drugs for different types of cancer and for patients with less advanced disease so dramatically increasing sales.

\begin{tabular}{llll}
\hline Trade name & Generic name & Manufacturer & Annual cost \\
\hline Herceptin & traztuzumab & Roche & $£ 50000$ \\
Mabthera & rituximab & Roche & $£ 40000$ \\
Glivec & imatinib & Novartis & $£ 50000$ \\
Erbitux & cetuximab & BMS/Merck & $£ 60000$ \\
Avastin & bevacizumab & Roche & $£ 70000$ \\
Tarceva & erlotinib & Roche & $£ 65000$ \\
Sutent & sunitinib & Pfizer & $£ 40000$ \\
Nexavar & sorafenib & Bayer & $£ 40000$ \\
Tykerb & lapatinib & GSK & not yet priced \\
Velcade & bortezimib & Bayer & $£ 30000$ \\
Panitumomab & ABX-EGF & Amgen & $£ 50000$ \\
Lucentis & ranibizumab & Genentech & $£ 60000$
\end{tabular}

approximate costs of their administration for one year are shown in Table 1.

In 2007 there will be at least six powerful new molecularly targeted anti-cancer drugs administered as simple tablets. It is unlikely the NHS will be able to afford any of them. In any case the NICE backlog will delay their assessment for at least a year. The availability of powerful tyrosine kinase inhibitors as straightforward tablets without the need for complex administration systems is bound to change the dynamic. Monoclonal antibodies are predicted to double the number of intravenous infusions required by 2011, stretching NHS delivery capacity to the limit. And personalized medicine - choosing the right therapy through genomics and proteomics - is likely to become available as an over the counter diagnostic service through emerging independent providers. These companies will almost certainly target a vast self-pay market as well as marketing their diagnostics to state and private insurers.

Radical radiotherapy is also becoming increasingly costly with the availability of conformal therapy techniques to allow a higher dose of radiation to be delivered to the tumour whilst sparing surrounding normal tissue. The more frequent use of conformal therapy will increase the costs of planning, simulation and delivery as the technical staff-time required becomes greater. Most new linear accelerator installations are equipped with multileaf collimators for intensity modulated radiotherapy. Furthermore the availability of real time verification systems and image guided radiotherapy will further increase the time and therefore cost of radical radiotherapy. The costs of radiotherapy will also increase as positron emission tomography-computed tomography (PET-CT) and other imaging techniques are used not only to plan radical treatment but also to monitor tumour destruction during therapy. Brachytherapy - the use of disposable radioiodine seed implants - for prostate cancer is getting much visibility on patient advocacy websites. Whilst this technique may allow a more precise and quicker way to deliver radical therapy its costs are higher and it is only available in certain centres that are beginning to market themselves aggressively in the private marketplace. Direct to consumer advertorials for such techniques are moving across the Atlantic in men's magazines. These will significantly increase demand.

Other cost pressures will come from increased use of new diagnostics, both in vitro and imaging, together with more frequent tumour response monitoring. Gene expression analyses are now allowing prognostic classification of lung, breast and colorectal cancer. Commercially available systems are being marketed at a cost of around $£ 2000$ per test. This is huge increase to the usual histopathology fee of around $£ 100$ per sample. PET-CT is growing in popularity: 20 new centres will be created over the next 12 months. PET-CT allows correlation of abnormal CT scan appearances with metabolic function of abnormal areas. Such functional images are useful in staging lung cancer and lymphomas and for monitoring therapy response. At a cost of $£ 2000$ per request, some limitation to the number of investigations per patient will be necessary. Risk banding for cancer based on the identification of polymorphisms in low penetrance cancer genes is likely to be offered by commercial providers during 2007, initially in London. The first consultation and tests will be around $£ 2000$ but the financial consequences in terms of increased screening and imaging costs could be 
much more significant. Screening could, for example, include a yearly breast MRI.

There are likely to be a significant number of new independent sector cancer providers competing for both NHS and insurance business. These will unblock the current under-capacity in cancer care delivery but drive up costs as quality and access improve. It is unlikely that the NHS will be able to afford the same standard of cancer care as received by patients in the US or Western Europe. This will make private medical insurance more attractive, provided that some product differentiation in the availability of diagnostics, radiotherapy and drugs as well as speed of access and consultant contact can be maintained.

How will UK pharma marketing companies react when patients just bypass their traditional strategies of selling to doctors, other health professionals and the NHS bureaucracy by ordering drugs through global internet pharmacies? We live, after all, in a consumer world. Patient information flow will become a far more powerful marketing tool than taking doctors out for free lunches or to conferences at exotic locations. Direct to consumer marketing using advertisements and subtle public relations activities to generate positive press stories are the increasingly used tools of the pharmaceutical and medical device industry. Roche was widely criticized recently for creating CancerUnited - a supposed cancer charity to lobby for better cancer care in Europe but run by their own PR agency and funded exclusively by themselves. Three MEPs resigned from CancerUnited's Board saying they had been duped. Pfizer have hired the very smart Coca Cola branding agency Edelman to advise on its cancer portfolio, and some time ago AstraZeneca bought Salick Health Care (now Aptium Oncology), a series of cancer centres in the US, in part to better understand doctors' prescribing habits. A new ethical framework in which to operate is urgently needed.

We are now leaving an economic Garden of Eden. How much we are willing to pay for an extra year of good quality life with cancer is going to be a key question for the babyboomer generation. And how will we allow individuals to contribute to their care in an equitable way? Consumerism and social solidarity do not sit comfortably together. A recent survey by the information charity Cancerbackup showed that $67 \%$ will vote in the next election on the basis of health policy and for $76 \%$ of us cancer is the most important health issue. ${ }^{5}$ No politician can afford to ignore these statistics. Yet we are impinging on the very core of NHS doctrine: care given freely on the basis of medical need and not ability to pay. Its high priests are rightly becoming very worried. For the sake of our patients we need to discuss these issues openly. There are no right or wrong answers but there is one certainty: we can never return to an age of innocence in the sacred garden.

Competing interests $\mathrm{KS}$ is a member of the steering committee of Doctors for Reform (www.doctorsforreform.com).

\section{REFERENCES}

1 NERA. Mind the Gap - the Future Funding of the NHS. London: NERA, 2006

2 Bosanquet N, Sikora K. The Economics of Cancer Care. Cambridge: Cambridge University Press, 2006

3 Wilking N, Johnson B. A Pan-European Comparison Regarding the Availability of Cancer Drugs. Stockholm: Karolinska Institutet, 2006

4 Bentzer S, Heeren G, Cottier B, et al. Towards evidence based guidelines for radiotherapy infrastructure: the ESTRO QUARTS project. Radiother Oncol 2005;75:355-63

5 Cancerbackup. Cancer a Public Priority? Attitudes Towards Cancer in Britain. London: Cancerbackup, 2006 